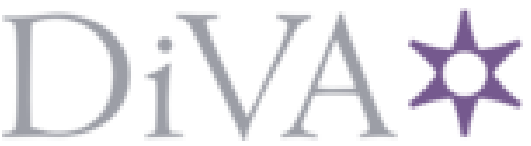

http://www.diva-portal.org

Preprint

This is the submitted version of a paper presented at IEEE GCCE2O18 (2018 IEEE 7th Global Conference on Consumer Electronics).

Citation for the original published paper:

Hayashi, M. (2018)

Neurodrug: Slapstick Effects by Destroying Neurons in a Neural Network

In:

N.B. When citing this work, cite the original published paper.

Permanent link to this version:

http://urn.kb.se/resolve?urn=urn:nbn:se:uu:diva-368984 


\title{
Neurodrug: Slapstick Effects by Destroying Neurons in a Neural Network
}

\author{
Masaki Hayashi \\ Department of Game Design \\ Uppsala University \\ Visby, Sweden \\ masaki.hayashi@speldesign.uu.se
}

\begin{abstract}
We propose a method to possibly generate slapstick effects by deliberately cutting out, tampering and re-connecting neurons in the neural network which has been trained in a proper way. We have developed two applications of a drumbeat generator and a CG character animation as the experiments to try out the idea. The results look interesting and show a certain potential for entertainment and also a viable way of interactive art.
\end{abstract}

Keywords-Neural network, deep learning, CG animation, drumbeat, slapstick

\section{INTRODUCTION}

Neural Network (NN) is relatively an old technology however, it has been widely studied for the use in deep learning which is the basis of recent Artificial Intelligence (AI) having achieved remarkable progress. Basically, an NN achieves problem-solving by teaching a neurons' network using a large amount of teaching data.

In this research, we propose a method using $\mathrm{NN}$ in an unusual way. We observe how the output of an $\mathrm{NN}$ behaves when the NN is artificially destroyed, such as disconnecting neurons, tampering sigmoid functions, re-connecting networks, etc. We call this operation "Neurodrug". It is apparent that if the correctly trained NN is destroyed, the output does not give the correct result. However, we hope that this may produce another kind of effect in a certain field of application such as entertainment. And that is the motivation of our research and development.

We conducted two experiments for the idea this time. One is to make an $\mathrm{NN}$ learn drumbeat in music, then by applying the manipulation of the $\mathrm{NN}$ and to observe the unexpected change of the beat pattern. The other is to create a Computer Graphics (CG) scene where six characters are talking each other and to make an NN learn which character looks at which, then we observe the characters' behavior when the manipulation is applied to the NN. Both are expected to have an effect close to "slapstick" rather than a serious effect.

We do not know this attempt could yield any practical or useful results, although it might be usable for automatic generation of slapstick play to make people laugh. Otherwise, it may function as a piece of art. Either way, this research has just begun, and in this article, we would like to introduce the working applications anyway.

\section{METHOD}

Figure 1 shows our method. First, an NN is trained by feeding reasonable teaching data. The output of this NN likewise produces reasonable behavior. While the $\mathrm{NN}$ is working, neurons in the $\mathrm{NN}$ are manipulated in various forms such as: to cut out the neuron's output, to change the influence of a neuron by multiplying a coefficient of -1 to +1 , to exchange the neuron's

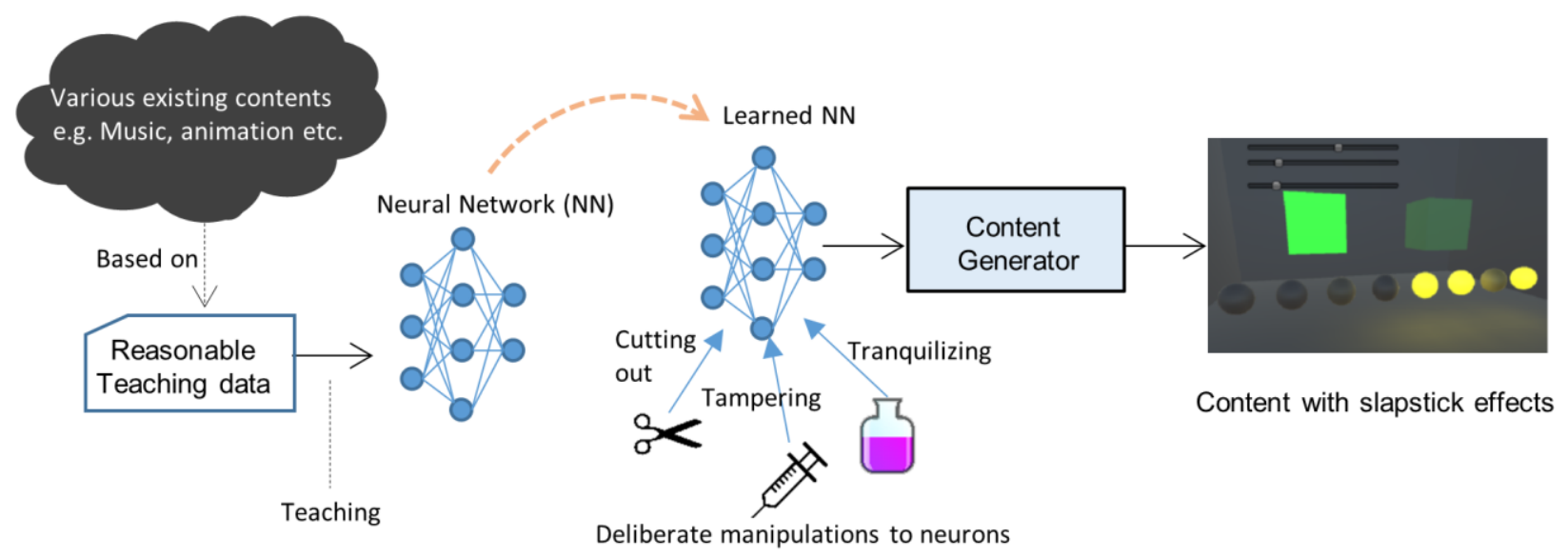

Figure 1: Method of Neurodrug 
connections, and so forth. In analogy, this would be equivalent to performing a surgical operation on the brain, inserting an electrode, paralyzing the work of the nerve by giving drugs. This operation should be made in real-time so that the results caused by those operations can be seen on the spot.

\section{EXPERIMENTS}

\section{A. Drumbeat generator}

In this experiment, a simple quadruple drum pattern is used as teaching data for two NNs. One has 2 inputs, 12 units in 1 hidden layer and 8 outputs for obtaining the beat number by giving sine and cosine of the beat rotation, and the other has 8 inputs, 12 units in 1 hidden layer and 2 outputs for obtaining the bass drum and the snare drum by giving the beat number. For the manipulation of the NN, it is applied to the hidden layers whose neurons' outputs are multiplied by a coefficient of -1 to +1 where you can change the location and area of range over the 12 neurons. The entire application is built on the Unity Game Engine.

Figure 2 shows the result. It makes the sound of a drum pattern with sampling sounds of the bass drum and snare drum. It also visualizes the beat and the drum stroke with CG (green: bass and snare, yellow: beat number). By operating the sliders, you can manipulate the NN in real-time. As you move the sliders, the pattern changes variously. It is hard to predict what pattern comes out but fun to play with. You can see the video here: https://youtu.be/d0YMIwau8Vg

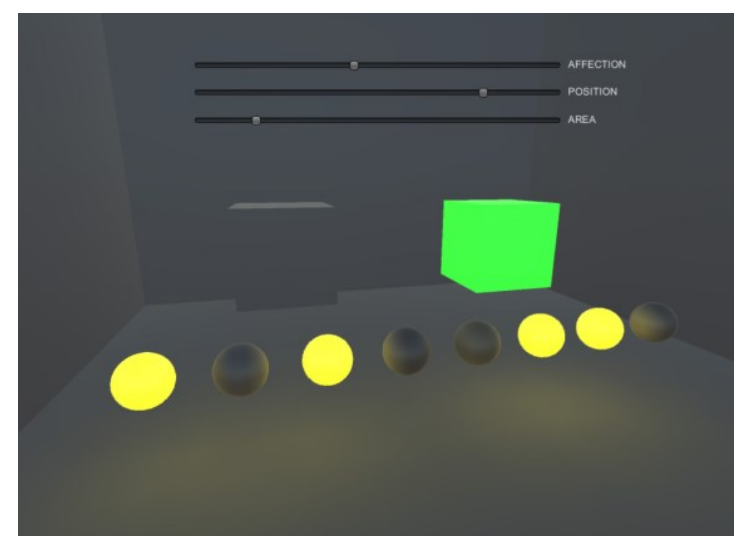

Figure 2: Drumbeat generator making sound of bass and snare drums with visualization.

\section{B. CG character behavior}

In this experiment, an application is made with six CG characters appear in a scene speaking dialogues by speech synthesis as shown in Fig. 3. The application is also built on Unity with TVML (TV program Making Language) [1] engine. The NN decides where each character looks at. Each character's "look at" is divided into three kinds: looking at a speaker, looking at another listener, looking down without seeing anyone. The teaching data is made based on the above and is fed to the $\mathrm{NN}$ for training. The NN has 13 units for the input layer, 12 units in 1 hidden layer and 6 units for the output layer. For input layer, information of the speaker and "look at" and elapsed time of the

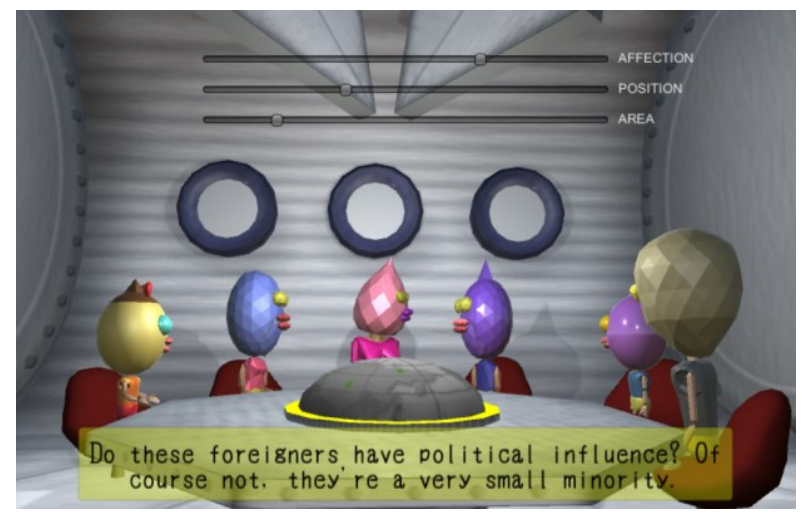

Figure 3: CG character animation. NN decides where each character looks at.

current "look at" are assigned to the 13 units and the output gives the next "look at".

Like in section 3.1, the 12 neurons in the hidden layer can be affected by the manipulation in real-time with the sliders. As you move the sliders, the character movements change accordingly, sometimes all the characters become random looking points or otherwise no change, etc. It is interesting to see the change in the output reacting immediately by the sliders, so it may be more meaningful in terms of the interactive art piece. You can see the video here: https://youtu.be/PBYala66wzo

\section{$I V$. DISCUSSION}

The point of our attempt is to try out our idea and to see what happens by actually implementing this crazy idea on working applications. Even with the drumbeat or the $\mathrm{CG}$ character control, there are many ways to implement the $\mathrm{NN}$, and for that part, more trial and error would be needed. There is no correct answer as to what to input to the input layer of the $\mathrm{NN}$, what to assign to the output layer, how to decide the number of hidden layers and the number of the unit. Also, what causes manipulations on $\mathrm{NN}$ to change the output behavior depends on how NN is configured. As a whole, it has not yet gone beyond trial and error for the moment.

According to the Wikipedia: "Slapstick is a style of humor involving exaggerated physical activity which exceeds the boundaries of normal physical comedy". Our idea could be regarded as an attempt trying to exceed the physical boundaries of normality by destroying the neurons in the NN. There is no clear reasoning other than that for the moment. However, during the process, we might find any relationship between slapstick and Neurodrug. Until then, rather than science or engineering, it might be natural that this attempt is viewed as a kind of "Interactive Art".

We now plan to increase the number of applications, change the configuration of NN variously, and repeat the experiments to dig this idea more.

\section{REFERENCES}

[1] M. Hayashi et al.: "T2V: New Technology of Converting Text to CG Animation", ITE Transactions on MTA, Vol.2, No.1, pp.74-82 (2014) 